\title{
Wozu Philosophische Anthropologie?
}

\author{
Von OLIVIA MITSCHERLICH (St. Gallen)
}

JOACHIM FISCHER: PHILOSOPHISCHE ANTHROPOLOGIE. Eine Denkrichtung des 20. Jahrhunderts. Karl Alber Verlag, Freiburg 2008, 684 S.

Gut zehn Jahre hat sich Joachim Fischer Zeit gelassen, um seine Studie über die Philosophische Anthropologie, die er 1997 in Göttingen als Dissertationsschrift eingereicht hat, als Buch zu veröffentlichen. Bisher war man (natürlich abhängig vom eigenen Wohnort) auf Fernleihe angewiesen; es sei denn, man hatte das Glück, von irgendjemandem die Kopie einer Kopie zu ergattern. Bemerkenswert ist, dass viele vor diesen umständlichen Beschaffungsprozeduren nicht zurückgeschreckt sind. Vielmehr wird Fischers These von der „Philosophischen Anthropologie“" als einem einheitlichen Denkansatz seit Jahren diskutiert. Dies liegt unter anderem auch an Fischers akademischer Präsenz: Nicht nur hat er 1999 die HelmuthPlessner-Gesellschaft mitbegründet und bis 2005 mit großem persönlichen Einsatz als deren erster Generalsekretär fungiert, auch hat er innerhalb und außerhalb der Plessner- und SchelerGesellschaften ein reges Engagement für die Sache der „Philosophischen Anthropologie“ und deren Hauptautoren entwickelt: zahlreiche Vorträge gehalten, Tagungen organisiert, Aufsätze veröffentlicht, Sammelbände herausgegeben.

Der Anspruch, dem Fischer mit seiner Studie genügen will, ist hoch. Er hat es sich zur Aufgabe gemacht, nachzuweisen, dass es sich bei der „Philosophischen Anthropologie“ nicht allein um die philosophische Beschäftigung mit den Phänomenen des Menschseins, sondern vielmehr um einen eigenständigen Denkansatz handelt, der über ein ,kategoriales Bildungsprinzip“ verfügt, ,das Erfahrungen in spezifischer Weise übersetzt und organisiert“ (15). Um seine These von der „Philosophischen Anthropologie“ als einem eigenständigen Denkansatz auszuweisen, geht Fischer sowohl wissenssoziologisch (er selbst sagt: realgeschichtlich) als auch philosophiegeschichtlich vor. Der erste Teil seiner Studie widmet sich der wissenssozio- 
logischen Frage, wie es zur Ausbildung einer Schule namens „Philosophische Anthropologie“ gekommen ist. Der zweite Teil ist auf die Frage nach dem systematischen „Identitätskern“ der „Philosophischen Anthropologie“" ausgerichtet.

Quantitativ gesehen legt Fischer auf den ersten Teil seiner Studie - der vier Fünftel des Buchs ausfüllt - den Schwerpunkt seiner Argumentation. In seinem wissenssoziologischen Versuch zu rekonstruieren, wie sich in den Interaktionen von Max Scheler, Helmuth Plessner, Arnold Gehlen, Erich Rothacker und Adolf Portmann eine eigenständige philosophische Schule herausgebildet hat, ist Fischer mit dem Problem konfrontiert, dass die genannten Autoren sich nur selten affirmativ aufeinander bezogen oder gar zusammengearbeitet haben. Zum Teil bestand kein Kontakt, zum Teil waren die Beziehungen durch Spannungen persönlicher, akademischer und politischer Natur stark belastet. Während der Zeit des Nationalsozialismus tun sich im Politischen unüberbrückbare Gräben auf: Portmann war Schweizer, Plessner wurde als so genannter „Halbjude“ ins Exil getrieben, Gehlen und Rothacker waren Parteimitglieder. Anders gelagert, aber ebenfalls grundlegend war das Zerwürfnis zwischen Scheler und Plessner im Streit um die Urheberschaft des Projekts der „Philosophischen Anthropologie“, der 1928 im Plagiatsvorwurf Schelers an Plessner gipfelt. Ein gutes Jahrzehnt später unterlässt Gehlen in seinem anthropologischen Hauptwerk Der Mensch jeden Verweis auf die Urheberschaft der „Philosophischen Anthropologie“ seitens des inzwischen verstorbenen Schelers und des nach Holland immigrierten Plessners - und handelt sich nun dadurch von Plessner den Vorwurf des Plagiats ein.

Fischer entschärft alle diese Spannungen in Hinblick auf die gemeinsame Sache: Die Autoren hätten in Bezug auf das philosophisch Gebotene die Zeichen der Zeit gleich interpretiert, gleich beantwortet und um diese theoretische Verbundenheit auch gewusst. Die Schule der „Philosophischen Anthropologie“ habe sich über die persönlichen Spannungen hinweg konstituiert. Fischer vertritt die These, dass die „Philosophische Anthropologie“ wissenssoziologisch eine „,Denk-Schule“ mit direkt und indirekt ,existierendem Kommunikationsnetz"“ darstelle (21 f.). So sei der akademische Wettstreit zwischen Scheler und Plessner genauso als eine Art der Kommunikation zu verstehen wie die Beziehungen zwischen den Autoren, die über die Vermittlung Dritter stattgefunden haben. Unter der indirekten, durch Dritte vermittelten Kommunikation versteht Fischer die parallel verlaufende Rezeption einschlägiger Arbeiten insbesondere aus der zeitgenössischen Biologie (zum Beispiel Uexküll und Köhler), gemeinsame persönliche Beziehungen (zum Beispiel von Scheler und Plessner zu Nicolai Hartmann) oder schließlich auch gemeinsame philosophische Gegnerschaften (vor allem zu Martin Heidegger). Fischer setzt solcherart ein sehr weites und in sich differenziertes Verständnis von Kommunikation an, um das Bestehen der „Denk-Schule“ der „Philosophischen Anthropologie“ zwischen 1919 und 1975 zu rekonstruieren.

Die Anfänge findet er in der Zeit, in der Scheler und Plessner an der neugegründeten Kölner Universität tätig waren und gemeinsam mit vielen Autoren aus der empirischen Biologie und Philosophie (etwa Viktor von Weizsäcker, Frederik Jacobus Johannes Buytendijk) zusammentrafen (vgl. 23 ff.). Das Ende der „Philosophischen Anthropologie“ markiert für Fischer ein Sammelband aus dem Jahre 1975 zu Ehren von Schelers 100. Geburtstag, in dem sowohl Plessner als auch Gehlen Rückblicke auf die Denkrichtung der „Philosophischen Anthropologie“" verfassen (450 ff.). Wenig später stirbt Gehlen und Plessner zieht sich aus der publizistischen Öffentlichkeit zurück. Zu diesem Zeitpunkt war es, wie Fischer zeigt, den Vertretern der „Philosophischen Anthropologie“ nicht gelungen, sich als Denkrichtung institutionell zu verankern. Nach der Emeritierung von Rothacker 1954 war kein philosophischer Lehrstuhl mehr mit einem Vertreter der „Philosophischen Anthropologie“ besetzt, es gab keine Zeitschrift, die sich der „Philosophischen Anthropologie“ verschrieben hätte, kein gemeinsames 
öffentliches Auftreten als Vertreter einer philosophischen Denkrichtung. So hatte die „Philosophische Anthropologie" den in den siebziger Jahren dominierenden philosophischen Ansätzen der „Kritischen Theorie“, der Hermeneutik und der aufkommenden Sprachphilosophie institutionell nichts entgegenzusetzen und ist bald der Historisierung (zum Beispiel bei Walter Schulz) verfallen beziehungsweise in der hermeneutischen Rezeption (durch Gadamer und Bollnow) um den Stachel des naturphilosophischen Vorgehens gebracht worden. Zwischen Anfang und Ende arbeitet Fischer sehr detailliert das Auf und Ab von Perioden der Krise (zum Beispiel nach Schelers Tod 1928) und des Neuanfangs (zum Beispiel mit den Veröffentlichungen von Rothackers Geschichtsphilosophie, Gehlens Der Mensch, Plessners Lachen und Weinen und Portmanns Tiergestalt Ende der dreißiger und Anfang der vierziger Jahre) heraus.

Um zum „Identitätskern“ der „Philosophischen Anthropologie“ vorzudringen, unterscheidet Fischer im zweiten, philosophiegeschichtlich angelegten Teil seiner Studie diesen Denkansatz von der philosophischen Beschäftigung mit dem Menschsein. Er arbeitet hierfür mit der terminologischen Unterscheidung von ,philosophischer Anthropologie“ und „Philosophischer Anthropologie“. Zwar ist Fischer nicht Urheber dieser Unterscheidung - sie findet sich unter anderem bereits bei Helmuth Plessner ${ }^{3}$-, aber es war Fischer, der sie prominent in die gegenwärtige Diskussion eingebracht hat. ${ }^{4}$ Die ,,philosophische Anthropologie“ steht für eine philosophische Subdisziplin, die sich über ihren Gegenstand - den Menschen - bestimme und deswegen neben philosophischen Überlegungen über andere Gegenstände - etwa dem Schönen oder dem Guten - rangieren könne. Sie ist philosophische Reflexion über den Menschen. Demgegenüber geht der Denkansatz der „Philosophischen Anthropologie“, dem Fischers eigentliches Interesse gilt, nicht in Bestimmungen des Erkenntnisgegenstandes „Mensch“ auf, sondern bestimmt sich über eine spezifische philosophische Einstellung, ein ,kategoriales Bildungsprinzip“. Sie nimmt den Menschen als Fundament ihrer philosophischen Erkenntnis in Anspruch.

Disziplin und Denkansatz seien unter anderem deswegen so schwer voneinander zu unterscheiden, weil sie sich zeitgleich herausgebildet hätten (482 ff.). Im Laufe des 19. Jahrhunderts untergraben die aufkommenden Wissenschaften der Ethnologie und der empirischen Biologie nach Fischer - der hier Plessners Verspäteter Nation folgt - das selbstverständliche Vertrauen ins Menschsein. Angesichts dieses Problematischwerdens des Menschseins entwickele sich die Anthropologie zu Beginn des 20. Jahrhunderts in Rückbezug auf die Philosophiegeschichte zu einer autonomen philosophischen Subdisziplin (vgl. 484). Vertreter dieser bis in unsere Gegenwart hineinreichenden Disziplin findet Fischer im breiten Spektrum von Freud über Cassirer, Heidegger, Sartre, Merleau-Ponty bis hin zu Mead und Dewey (485 f.).

Einhergehend mit dieser Disziplinausbildung, in sie verwoben und von ihr verdeckt, formiert sich nach Fischer der Denkansatz der „Philosophischen Anthropologie“. Die Gründungsschriften dieses Denkansatzes findet Fischer in den Schriften Die Stellung des Menschen im Kosmos von Max Scheler und Die Stufen des Organischen und der Mensch von Helmuth Plessner, die 1927 beziehungsweise 1928 erschienen sind (vgl. 487). Mit diesem neuen Denkansatz reagieren die Vertreter der „Philosophischen Anthropologie“" Fischer zufolge auf die Krise, in der sich die Philosophie selbst zu Beginn des 20. Jahrhunderts befunden hat (vgl.

3 Vgl. zum Beispiel H. Plessner, Die Aufgabe der Philosophischen Anthropologie [1937], in: ders., Gesammelte Schriften, Bd. VIII, Frankfurt/M. 1983, 33-51.

4 So hat etwa Hans-Peter Krüger die obige Unterscheidung mit Bezug auf Fischer aufgenommen, um dann allerdings - gegen Fischer - Plessner als alleinigen Begründer des Denkansatzes der „Philosophischen Anthropologie“ zu behaupten (vgl. H.-P. Krüger, Zwischen Lachen und Weinen, Bd. I, Berlin 1999, 24). Weniger beachtet als bei Fischer findet sich die Unterscheidung auch schon bei Herbert Schnädelbach (vgl. H. Schnädelbach, Philosophie in Deutschland. 1831-1933, Frankfurt/M. 1983, 265-273). 
507 ff.). Im Laufe des 19. Jahrhunderts hat das Vernunftparadigma nach Fischer - der sich hier abermals an Plessners Verspäteter Nation orientiert - seine selbstverständliche Glaubwürdigkeit verloren. Die Vernunft wird nicht mehr als letzter Sinnhorizont anerkannt, in dem sich alles Denken und Handeln vorfindet, sondern in ihrer Bedingtheit durch die übergreifenden Wirklichkeitszusammenhänge des biologischen und sozialen Lebens aufgedeckt. Auf Grund dieser Anthropologisierung und Soziologisierung verliert der „Deutsche Idealismus“, der nach Fischer den seit 1800 vorherrschenden Denkansatz dargestellt hat, das ihn tragende Fundament. Seine Erbschaft tritt in Fischers Darstellung der neue Denkansatz der „Philosophischen Anthropologie“ an (vgl. 515 ff.). Dessen Vertreter eigneten sich das moderne Wissen vom biologischen und sozialen Leben an, um unter den Bedingungen moderner Wissenschaft an der Intuition des „Deutschen Idealismus“ festzuhalten, dass die Vernunft beziehungsweise der Geist autonom und fähig zur Selbstvergewisserung sei (vgl. 515 f.). Gemeinsam sei den Vertretern der „Philosophischen Anthropologie“ nun nicht nur dieses programmatische Ziel, sondern auch dessen Bewältigung: Zur Verteidigung der Vernunftautonomie werde nicht bei den Vernunftgesetzen, sondern grundlegender bei den Gesetzmäßigkeiten des biologischen Lebens angesetzt, um von dort aus zu der Selbstgesetzgebung der Vernunft vorzudringen. Diese Denkbewegung vom biologischen Leben zum Geist in seiner Eigenständigkeit stellt nun Fischer zufolge den „Identitätskern“ dar, der die „Philosophische Anthropologie“ zu einen einheitlichen Denkansatz zusammenschweißt.

Im Ausgang von diesem „Identitätskern“ erschließt sich Fischer eine Reihe von Charakteristika, die den Denkansatz der „Philosophischen Anthropologie“ auszeichnen sollen (vgl. 520 ff.). Alle Vertreter der „Philosophischen Anthropologie“ stellten sich in Bezug auf das Subjekt-Objekt-Verhältnis nicht in die Sphäre der Subjektivität, sondern gingen vom Objekt aus. Zur Bestimmung des Objektpols gingen sie nicht vom menschlichen, sondern basaler vom lebendigen Sein aus. Das lebendige Sein wiederum verstünden sie (in Anschluss an Uexküll) als Funktionskreis, der von dem Ineinandergreifen von Organbeschaffenheit und Umwelt gebildet wird. Methodisch verschafften sich die Denker der „Philosophischen Anthropologie“ auf diese Weise die Möglichkeit, einen „flankierenden Blick“ auf die menschliche Subjektivität zu werfen (522). Von der methodischen Anlage her sieht Fischer damit bei allen Vertretern der „Philosophischen Anthropologie“ einen Primat der Naturphilosophie vor der Kultur-, Geschichts- oder Sozialphilosophie. Zwar werde Künstlichkeit und Sozialität des Menschen thematisiert, das geschehe jedoch im Ausgang von der menschlichen Natur (549 f.).

Fischers Studie provoziert meiner Ansicht nach eine Reihe von Nachfragen. Zunächst sei ein systematisches Problem vermerkt. Der Ansatz der „Philosophischen Anthropologie“, den Fischer rekonstruiert, muss an seiner Selbstanwendung scheitern. Wenn menschliches Sein dadurch ausgezeichnet ist, dass das natürliche Ineinandergreifen von Umwelt und Organismus durchbrochen und menschliches Denken und Handeln an einen künstlichen Sinnhorizont verwiesen ist, dann ist damit einem philosophischen Ansatz der Boden entzogen, der einseitig den Primat einer Naturphilosophie vertritt und die menschliche Natur als Wahrheitsfundament philosophischer Erkenntnis in Anspruch nehmen will. Da Fischer selbst explizit auf eine systematische Diskussion der Tragfähigkeit des von ihm vorgestellten Ansatzes verzichtet, soll der Schwerpunkt meiner Nachfragen im Folgenden jedoch den von ihm behaupteten „Identitätskern“ der „Philosophischen Anthropologie“ betreffen (vgl. 15 ff.).

Wiederholt - zuletzt von Jens Hacke in seiner Rezension für die Süddeutsche Zeitung ${ }^{5}$ ist gegen Fischer eingewandt worden, dass der von ihm herausgearbeitete „Identitätskern“

5 Vgl. J. Hacke, Suche nach Wirklichkeit. Eine Beziehungsgeschichte der Philosophischen Anthropologie, in: Süddeutsche Zeitung, 28. Juli 2008. 
der „Philosophischen Anthropologie“ zu vage sei, um eine wirkliche Einheit zwischen den Autoren zu begründen, und dass Fischer mithilfe dieses dünnen Einheitskriteriums die Unterschiede zwischen den vereinten Autoren unterschlage. ${ }^{6}$

Ich kann mich diesem Einwand nicht entziehen. Besonders deutlich zeigt sich dies an dem von Fischer nivellierten Unterschied zwischen den Philosophieverständnissen Schelers, Plessners und Gehlens. Auch wenn alle von Fischer versammelten Autoren unter anderem über eine Naturphilosophie verfügen, die ihren Anfang beim basalen biologischen Leben nimmt, um ,von unten“ zum menschlichen Geist durchzufragen, so unterscheiden sie sich doch grundsätzlich in der methodischen Anlage ihres Philosophierens.

Gehlen selbst reflektiert dies explizit, indem er seine „empirische Anthropologie“ von den „metaphysischen Anthropologien“ seiner Vorgänger abgrenzt. Hier kann es nun nicht überzeugen, wenn Fischer diesen prinzipiellen Unterschied durch die Behauptungen aufzuheben versucht, dass sich einerseits auch Scheler und Plessner positiv auf die empirischen Wissenschaften beziehen und „Philosophische Anthropologie [...] mit dieser Verarbeitung der verschiedenen positiven Wissenschaften auch bei Scheler und Plessner wie bei Gehlen, empirische Philosophie“" sei; und dass andererseits auch Gehlen den Gesichtspunkt zur Gesamtauffassung des Menschen nicht den empirischen Wissenschaften, sondern philosophischer Reflexion entnehme (555 f.). Mit diesem Versöhnungsversuch bewegt sich Fischer nämlich allein auf inhaltlicher und gerade nicht auf der von Gehlen beschrittenen methodischen Ebene der Bewährung der inhaltlichen Einsichten in ihrem Wahrheitsanspruch.

Noch offensichtlicher werden die von Fischer unterschlagenen Differenzen im Philosophieverständnis der drei genannten Autoren, wenn man sich anschaut, was in Fischers Darstellung fehlt - nämlich der Grund, warum Gehlen Scheler und Plessner zu Recht als Metaphysiker bezeichnet. Zwar erwähnt Fischer, dass Scheler seine „Philosophische Anthropologie“ in den umfassenden Zusammenhang seiner Metaphysikkonzeption integriert, er zieht daraus jedoch keine Schlüsse für den Status von Schelers Anthropologie. Fischers Auslassungen bei Plessners Werk sind mindestens ebenso grundlegend. Plessner ordnet seiner Naturphilosophie eine gleichberechtigte Kultur- beziehungsweise Geschichtsphilosophie bei. Durch diesen Doppelansatz nimmt Plessner nicht nur auf inhaltlicher, sondern auch auf methodischer Ebene ernst, dass Menschsein in natürlich-künstlicher Gebrochenheit stattfindet. Mit interpretatorischer Gewalt versucht Fischer, diese geschichtsphilosophische Dimension des Plessnerschen Denkens auszuklammern. Zum einen deutet er die zentrale Stelle in Plessners philosophischer Selbstverständigung, in der dieser die Gleichursprünglichkeit von Natur- und Kultur- beziehungsweise Geschichtsphilosophie entwickelt, in einen Primat der Naturphilosophie um. ${ }^{7}$

6 Vgl. auch M. Schloßberger, Die Ordnung des menschlichen Gefühlslebens, in: H.-P. Krüger u. G. Lindemann (Hg.), Philosophische Anthropologie im 21. Jahrhundert, Berlin 2006, 254-273, hier: $255 \mathrm{ff}$.

7 Vgl. H. Plessner, Die Stufen des Organischen und der Mensch, in: ders., Gesammelte Schriften, Bd. IV, Frankfurt/M. 1981, 70: „Vom Menschen als personaler Lebenseinheit ist also zunächst zu handeln und den mit ihr wesenskoexistenten Schichten des Daseins, des Seins überhaupt. [...] Wie weit reicht diese Wesenskoexistenz, und wo beginnt der Zufall? Diese Frage läßt sich in doppelter Richtung aufrollen, horizontal, d. h. in der Richtung, welche durch die von ihm gesuchte Beziehung des Menschen zur Welt in seinen Taten und Leiden festgelegt ist, und vertikal, d. h. in der Richtung, die sich aus seiner naturgewachsenen Stellung in der Welt als Organismus in der Reihe der Organismen ergibt. In diesen beiden Richtungen kann man hoffen, den Menschen als Subjekt-Objekt der Kultur und als Subjekt-Objekt der Natur wirklich zu umfassen, ohne ihn in künstlichen Abstraktionen aufzuteilen.“ (Hervorh. von mir; O. M.) Auf diese Textstelle bezieht sich Fischer, wenn er schreibt: „Nimmt man die Philosophische Anthropologie als Denkansatz, verlangt sie primär notwendig einen internen Bezug zu einer Biophilosophie, sekundär zur Kulturphilosophie. Es ist zwar 
Zum anderen deutet er die Schrift - Macht und menschliche Natur von 1931 -, in der Plessner seine Geschichtsphilosophie entwickelt, als Produkt einer (durch Schelers Plagiatsvorwurf ausgelösten) Lebenskrise, in der Plessner den Denkansatz der „Philosophischen Anthropologie“" aus den Augen verloren und den anders gelagerten hermeneutischen Denkansatz der Diltheyschule um Georg Misch übernommen habe (vgl. $117 \mathrm{ff}$.). Wenn man im Unterschied zu Fischer die Plessnersche Geschichtsphilosophie in ihrem Eigenrecht ernst nimmt, dann stellt sich das Plessnersche Denken der 20er und 30er Jahre als natur- und geschichtsphilosophischer Doppelansatz dar. ${ }^{8}$ Sein Denken zeigt sich dann als ein Ansatz negativer Metaphysik, der aus der natur- und geschichtsphilosophischen Gebrochenheit philosophiert und derart den Wahrheitsgrund der Wirklichkeit überhaupt offen hält.

Solch ein in sich gebrochenes Denken steht allerdings in grundsätzlichem Widerspruch zu Fischers Ansatz einer „Philosophischen Anthropologie“ mit deren Primat einer Naturphilosophie, der das menschliche Leben zum neuen Wahrheitsgrund der Philosophie erklärt. Plessners negative Metaphysik sprengt damit den von Fischer abgesteckten „Identitätskern“ der „Philosophischen Anthropologie“. Zwar behauptet Fischer, von Plessners Begriff der „exzentrischen Positionalität" auszugehen, seine verkürzte Darstellung vermittelt allerdings viel eher den Eindruck, dass er Scheler und Plessner ,gehlenisiert“ hat, um dann einen einheitlichen Denkansatz zu behaupten, der vor allem Gehlen trifft. Wenn man den Primat der Naturphilosophie bestreitet, bleibt als Gemeinsamkeit zwischen den genannten Autoren allein noch übrig, dass sie über eine von der modernen Biologie auf unterschiedliche Weise beeinflusste Naturphilosophie verfügen, die sie methodisch unterschiedlich anlegen.

Wenn man sich vor Augen führt, wie viele Anstrengungen Fischer leisten muss, um die unterschiedlichen Positionen seinem Denkansatz der „Philosophischen Anthropologie“ zu subsumieren, Widerstrebendes abzublenden, Differenzen einzuebnen, stellt sich die Frage nach dem „Wozu“. Diese Frage gewinnt zusätzlich an Gewicht, wenn man sich daran erinnert, dass die „Philosophische Anthropologie“ nach Fischer zu Beginn des 20. Jahrhunderts den „Deutschen Idealismus“ abgelöst haben soll, der seinerseits ab 1800 vorgeherrscht haben soll. Gab es denn je einen Denkansatz namens „Deutscher Idealismus“?? Auch scheint es nicht zufällig zu sein, dass man im Allgemeinen (Fischer bildet hier eine Ausnahme) nur dann zu den Schubladen des „Deutschen Idealismus“, des „Neukantianismus“, der „Philosophischen Anthropologie" oder wie sie nicht sonst alle beschriftet sein mögen, greift, wenn man sich von ihnen abgrenzen will, sich in Bezug auf das eigene Denken solcher Zuordnungen jedoch gerade verwehrt.

Genau an dieser Einteilung der Philosophie in verschiedene Schubladen will Fischer offensichtlich festhalten - und kämpft deswegen mit solcher Vehemenz für einen einheitlichen

richtig festgestellt worden, dass die Philosophische Anthropologie thematisch sich gleichermaßen auf zwei Vergleichsreihen bezieht - auf die ,vertikale' des Vergleichs der menschlichen Lebensform mit anderen organischen Lebensformen und auf die ,horizontale' des Vergleichs verschiedener differenter Soziokulturen untereinander. Methodisch aber, vom Ansatzpunkt her gibt es - bei allen fünf Autoren - einen Primat der vertikalen Untersuchungsrichtung gegenüber der horizontalen Richtung der Differenzen der Kulturen.“ (549) Wie Plessners philosophisches „Aufrollen“ nur thematisch und nicht methodisch verstanden werden kann, bleibt Fischers Geheimnis.

8 Ich habe diesen Ansatz zu entwickeln versucht in: O. Mitscherlich, Natur und Geschichte. Helmuth Plessners in sich gebrochene Lebensphilosophie, Berlin 2007.

9 Seit Jahren tritt Walter Jaeschke dafür ein, in der Philosophiegeschichte von der inhaltlichen Qualifizierung des „Deutschen Idealismus“ zur Epochenbezeichnung der „klassischen deutschen Philosophie“" zu wechseln, da zentrale Protagonisten wie Jacobi oder der späte Schelling keine Idealisten waren. 
Denkansatz der „Philosophischen Anthropologie“, der unter sich mehrere Vertreter fassen soll. „Ein Theorieprogramm in diesem Sinne kann auch nicht nur einen Bezugsautor haben, sondern muss [...] mehrere unabhängige Köpfe tangieren, die es miteinander teilen und mit ihm arbeiten, die es - ob in Konsens oder in Rivalität - kommunizieren. " (488) Dahinter steht ein am Paradigma der neuzeitlichen Wissenschaften ausgerichtetes Philosophieverständnis. Philosophie soll Forschung sein und entsprechende Ergebnisse erzielen. ${ }^{10} \mathrm{Ob}$ der Philosophie damit gedient ist, wenn sie auf dieses methodische Ideal verpflichtet ist, bleibt zweifelhaft; jedenfalls kann Fischer ein Forschungsprogramm der „Philosophischen Anthropologie“ nur identifizieren, indem er Unterschiede nivelliert. So ist es wohl auch nicht erstaunlich, dass Fischers Studie vor allem durch ihren ersten Teil - der wissenssoziologischen Aufarbeitung, wie die Bildung einer Schule namens „Philosophische Anthropologie“ stattgefunden hat - beeindruckt. Hier schöpft Fischer aus einem schier unbegrenzten Fundus von Forschungsergebnissen, die auf akribischer Archivarbeit fußen.

10 Vgl. 482 f.: „Die angestrebte [...] Klärung ist ein Angebot, mit dem philosophiegeschichtlichen Bestand der ,philosophischen Anthropologie“ / ,Philosophischen Anthropologie“ im 20. Jahrhundert philosophiesystematisch so umzugehen, dass er für philosophisch-anthropologische Forschungsprojekte im 21. Jahrhundert überhaupt anschlussfähig wird.“ 